\title{
Early detection of visual field progression in glaucoma: a comparison of PROGRESSOR and
}

STATPAC 2

\author{
Ananth C Viswanathan, Fred W Fitzke, Roger A Hitchings
}

\begin{abstract}
Aim-To compare the performance of PROGRESSOR (pointwise linear regression) and STATPAC 2 (comparison with baseline values) in detecting early deterioration in the visual fields of glaucoma patients.

Methods-Visual field series from 19 untreated normal tension glaucoma eyes which were deteriorating on clinical grounds were analysed by PROGRESSOR and STATPAC 2. Progression criteria for PROGRESSOR were (1) inner points: slope $<-1$ dB/year, $p<0.05$ and (2) edge points: slope $<-2 \mathrm{~dB} /$ year, $\mathrm{p}<0.05$. Criteria for STATPAC 2 were $p<0.05$ change probability for any point on three consecutive fields. Detection time was defined as the time interval between the initial field and the first field in which at least one progressing point was identified. Detection times produced by the two techniques were compared.
\end{abstract}

Results-PROGRESSOR and STATPAC 2 agreed on progression in all 19 eyes. Mean detection time for PROGRESSOR was 1.077 (SD 0.985 ) years and for STATPAC 2 was 2.161 (1.357) years. PROGRESSOR detected progression sooner than STATPAC 2 in 18 eyes ( $p<0.01$, Wilcoxon matched pairs signed rank test). PROGRESSOR detected progression earlier by a mean of $1.085(0.936)$ years.

Conclusions-PROGRESSOR consistently detected progression earlier than STATPAC 2 . The PROGRESSOr software is a useful tool for the early detection of visual field deterioration in glaucoma.

(Br f Ophthalmol 1997;81:1037-1042)

Institute of

Ophthalmology,

London EC1V 9EL

A C Viswanathan

F W Fitzke

Moorfields Eye Hospital, City Road, London EC1V 2PD

R A Hitchings

Correspondence to: Fred W Fitzke, PhD, Institute of Ophthalmology, 11-43 Bath Street, London EC1V 9EL.

Accepted for publication 28 April 1997
An important goal in the management of chronic glaucoma is the early, reliable detection of deterioration in the visual field. There is inherent 'noise' in the data obtained from serial automated field tests (long term fluctuation ${ }^{1}$ ): this fluctuation is greater in glaucoma patients than in normals. ${ }^{2}$ The criteria by which progression should be measured are a continuing subject of debate. ${ }^{3-8}$ Visual inspection of a series of fields and the use of clinical judgment are unreliable methods for diagnosing progression or stability, even when performed by experienced observers. ${ }^{9}$ For this reason, and because the results of automated perimetry invite numerical analysis, a number of methods to estimate change in glaucomatous visual fields have been developed.

One group of methods rely on estimates of change in summary measures of the field such as regression analysis of the mean defect value, ${ }^{10}$ mean deviation, ${ }^{11}$ other global measures, ${ }^{11}$ measurement of whole field and quadrantic sensitivity losses, ${ }^{12}$ and trend and regression analysis of various estimates of the sensitivity of the whole field or parts of it. ${ }^{13-15}$ However, the analysis of summary measures, whether based on the whole field or on clusters of points within it, has been found to be 'remarkably poor' ${ }^{16}$ and 'of little value'17 in detecting glaucomatous change. Summary measures largely or completely ignore the detailed spatial information contained within computerised field tests and are insensitive to early localised change.$^{18}$ Furthermore, different regions of the visual field may deteriorate at different rates. ${ }^{4} 1519$

A widely available software package for estimating deterioration in serial glaucomatous visual fields is the STATPAC 2 glaucoma change probability analysis ${ }^{20}$ for the Humphrey field analyser (Humphrey Instruments Inc, San Leandro, CA, USA). This software avoids the problems inherent in the methods discussed above by considering the field on a point by point basis. Two of the three initial fields are selected as a 'baseline' and subsequent fields are compared with this baseline in a pointwise manner. Points are labelled with a black triangle (Fig 1) if they are associated with a $p$ value of $<0.05$ for change against a reference database.

PROGRESSOR $^{21}$ is a software package which analyses visual field progression using pointwise linear regression of sensitivity on time. This technique has been used for several years to investigate glaucomatous visual field change $^{22}{ }^{23}$ and has recently been re-examined. ${ }^{11}$ PROGRESSOR produces a cumulative graphical output as shown in Figure 2A. Each test location is shown as a bar graph in which each bar represents one test. The length of the bar relates to the depth of the defect (longer bars represent lower sensitivities) and the colour of the bar relates to the $\mathrm{p}$ value of the regression slope (Fig 2B). The pointwise linear model has been demonstrated to provide a valid framework for detecting and forecasting glaucomatous loss. ${ }^{24}$

PROGRESSOR has been found to emulate STATPAC 2 closely in diagnosing individual locations in a single field of a series as either 

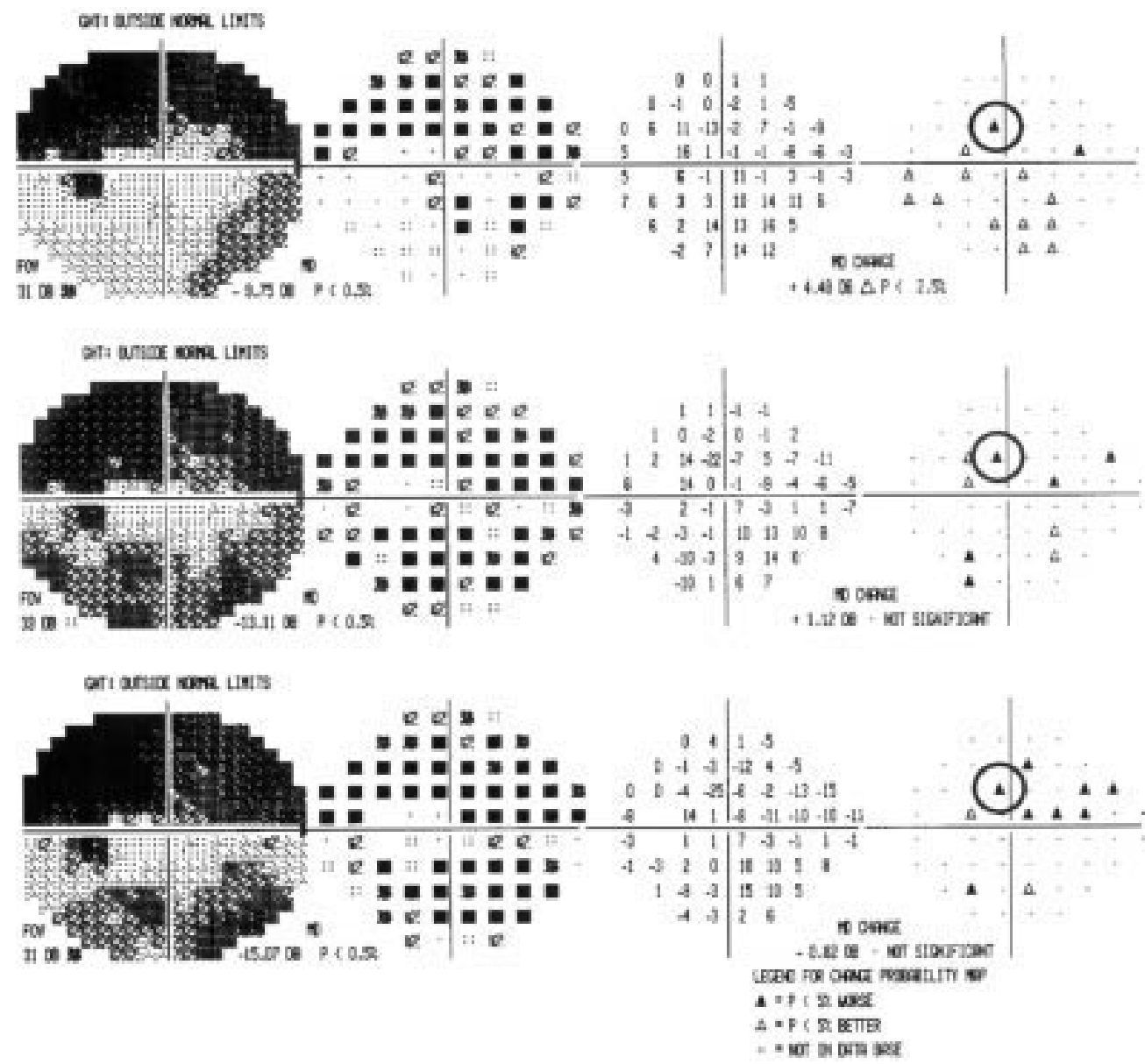

Figure 1 Example of a section of the STATPAC 2 glaucoma change probability analysis. The test location highlighted is labelled as showing significant deterioration in each of the three consecutive fields.

stable or progressing when the appropriate progression criteria are used. ${ }^{25}$ However, the relative ability of the two algorithms to detect the first sign of deterioration has not been investigated directly. This is an important aspect of the technique's behaviour to compare, since the timely and reliable detection of glaucomatous visual field progression is of paramount clinical importance: in the presence of adequate intraocular pressure control, questions concerning the commencement or alteration of therapy are prompted only when definite visual deterioration is seen.

\section{Methods}

SUBJECTS

The Moorfields Eye Hospital visual field database currently contains 64949 automated visual field records from 9482 patients. Records were selected for study on the basis of the following criteria:

(1) Untreated normal tension glaucoma patients were chosen as it was felt to be important to analyse the natural history of glaucomatous visual damage in the absence of the potentially confounding effects of medical therapy. A cohort of 220 such patients, whose diagnosis had been confirmed on phasing, was identified.

(2) Their visual fields showed progressive deterioration of a typically glaucomatous na- ture. This was done by inspection of the STATPAC overview analysis of serial Humphrey visual fields by an experienced observer.

(3) All subjects were experienced in Humphrey 30-2 tests and able to produce reliable computerised visual fields (less than 30\% fixation losses and false negatives and less than $15 \%$ false positives). Each had had at least two tests over 4 months before the observation period: this is sufficient to obviate any learning effects $^{2627}$ which may delay the diagnosis of progression.

(4) All subjects had visual acuity of $6 / 12$ or better. None had significant ocular pathology apart from normal tension glaucoma.

On the basis of the foregoing criteria, 19 eyes from 13 subjects were selected.

An indication of the degree of glaucomatous damage in the selected group is given by the following summary measures of the mean deviation (MD) of the initial field in each test series: the mean of the MDs was -6.81 (SD 6.01) $\mathrm{dB}$, the median was $-5.43 \mathrm{~dB}$, and the range was $-22.40 \mathrm{~dB}$ to $+1.07 \mathrm{~dB}$.

\section{TESTING STRATEGY}

All tests were performed on a standard Humphrey automated perimeter. The full threshold 30-2 program with standard 4-2 dB staircase strategy was used throughout. Tests 


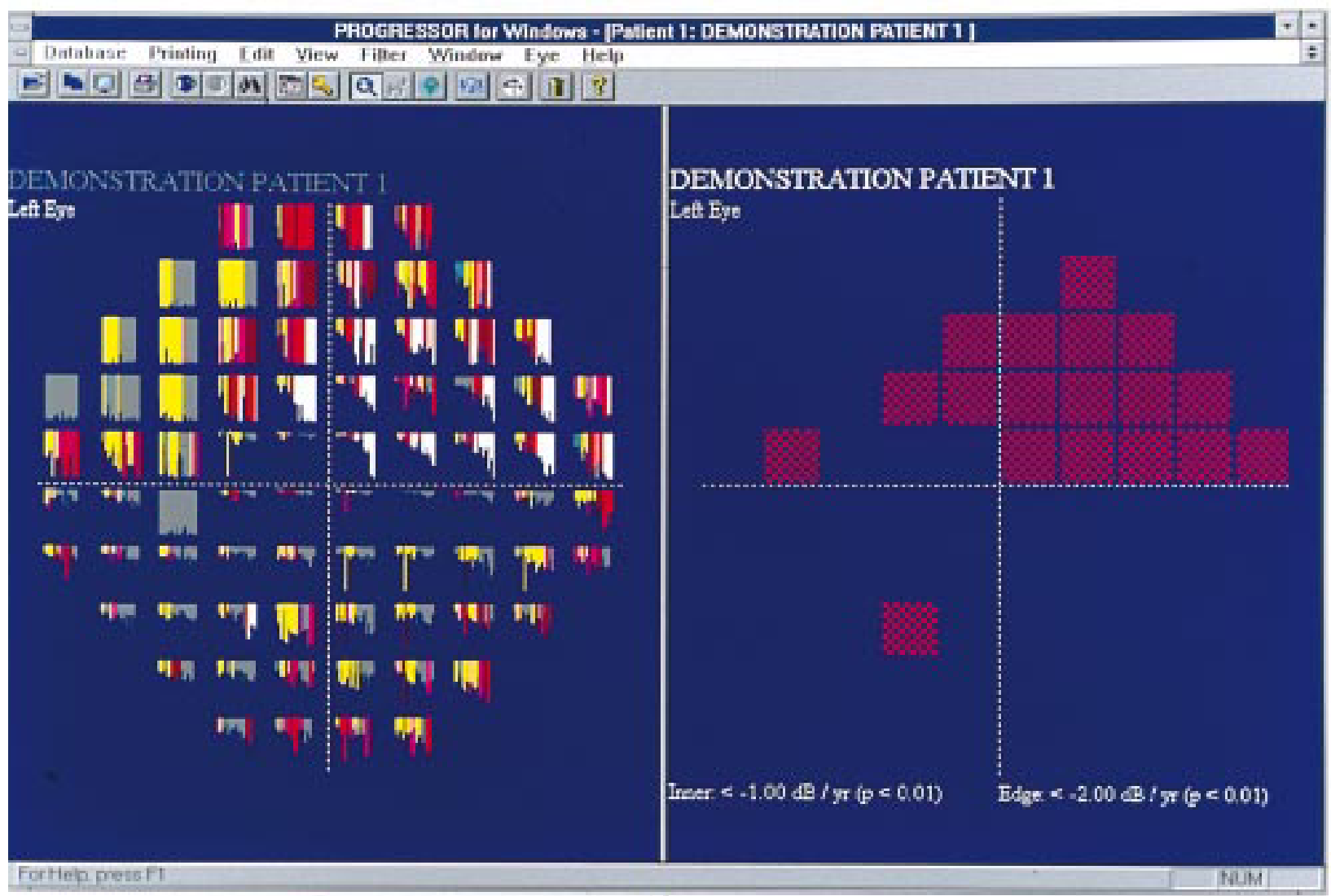

Figure 2 (A) Example of the cumulative graphical output of PROGRESSOR for Windows for the left eye of an untreated normal tension glaucoma patient with visual field progression. The left pane shows the bar graph output. The right pane shows the locations which satisfy the progression criteria.

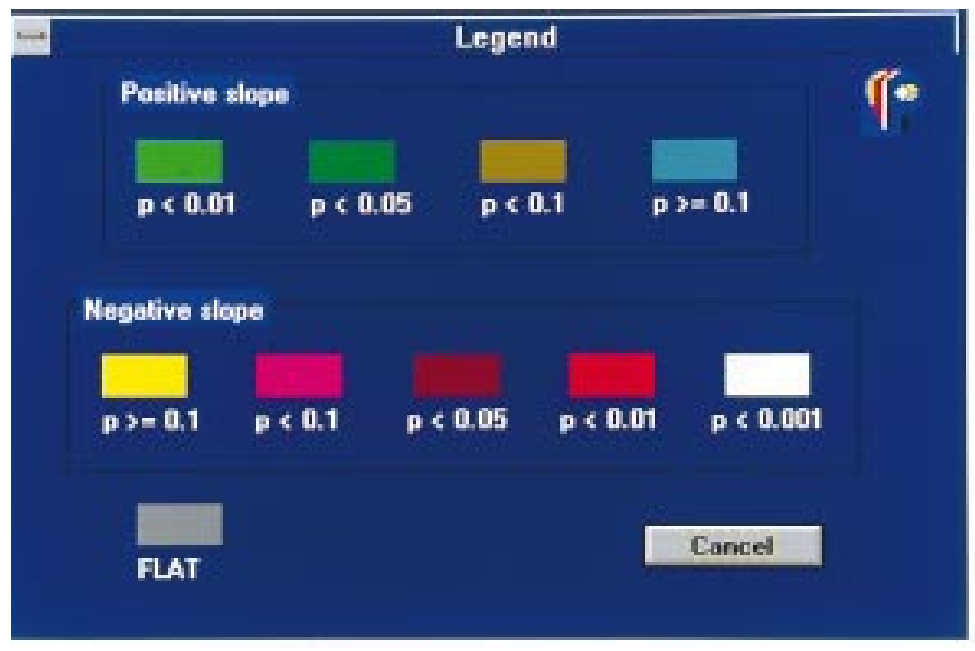

Figure 2 (B) Legend for PROGRESSOR for Windows.

were performed at intervals of approximately 4 months.

PROGRESSION CRITERIA

Glaucoma change probability: STATPAC 2

A field series was regarded as progressing if the glaucoma change probability analysis of STATPAC 2 marked any test location as showing significant deterioration $(\mathrm{p}<0.05)$ from the baseline relative to the Humphrey normal database on three consecutive occasions. The requirement for a location to be repeatedly ascribed a high probability of change over a series of con- secutive fields has been recommended by the originators of STATPAC $2^{20}$ and has been used in previous studies. ${ }^{21}{ }^{25}$ The specific criterion of three consecutive fields is currently in use in the Early Manifest Glaucoma Trial in Malmö, and the importance of confirmatory testing to establish progression relative to a baseline has been upheld in the ongoing collaborative normal tension glaucoma study. ${ }^{28}$

PROGRESSOR

A field series was regarded as progressing if PROGRESSOR identified at least one test location with a negative slope of $1 \mathrm{~dB}$ per year or worse associated with $\mathrm{p}<0.05$ for a two tailed $t$ test of the slope against zero (that is, the null hypothesis of no deterioration). The slope criterion of $1 \mathrm{~dB}$ per year represents a rate of sensitivity loss approximately 10 times greater than the normal age related decline. ${ }^{29}$ Edge points are known to be more subject to fluctuation ${ }^{29}$ so a stricter slope criterion of $2 \mathrm{~dB}$ per year (also with $\mathrm{p}<0.05$ ) was introduced for them. These slope criteria, in combination with a less stringent slope significance criterion of $p<0.1$, have been demonstrated to compare closely with the Humphrey STATPAC 2 glaucoma change probability analysis. ${ }^{2125}$

\section{DETECTION TIME}

The detection time for a given field series for a given algorithm was defined as the time interval between the initial field in the series 


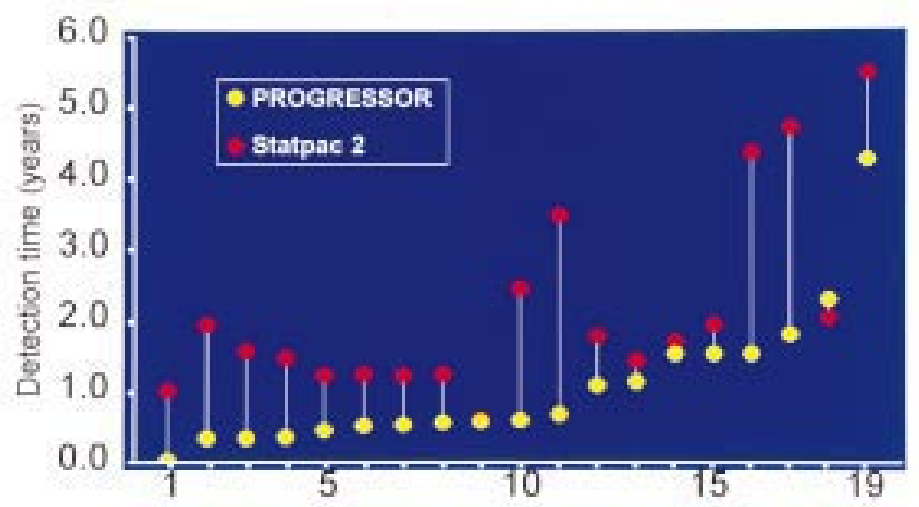

Field series ranked in order of detection time by PROGRESSOR

Figure 3 Drop line graph of detection times for each field series for PROGRESSOR and STATPAC 2. The field series are ranked in order of detection time by PROGRESSOR.

and the field when the progression criteria for that algorithm (see above) were first satisfied.

RELIABILITY OF EARLY DETECTION

There is at present no 'gold standard' for the identification of visual field progression in glaucoma. Thus, it is very difficult to assess whether a given technique is detecting 'true' progression. Some authors have used clinical impression against which to compare the performance of various algorithms, ${ }^{17}$ but this has been shown to be a largely subjective measure. ${ }^{9}$ Others have avoided the problem entirely by not attempting to estimate the reliability of their techniques. ${ }^{11}$

In the field series selected for this study, all three methods of assessing change (clinical expertise, PROGRESSOR, and STATPAC 2) agreed that all of the series showed progression. However, this study was designed to examine early detection of progression by the algorithms, so it was important to assess whether progression detected at a given field in a series was sustained in the rest of the series: it is likely that if progression is diagnosed in one field but not in subsequent fields the diagnosis is incorrect.

The reliability of early detection of progression by PROGRESSOR and STATPAC 2 was assessed by examining whether at least one of the locations initially detected as progressing was still progressing in the final field of the series. For STATPAC 2, the criterion of repeatability over

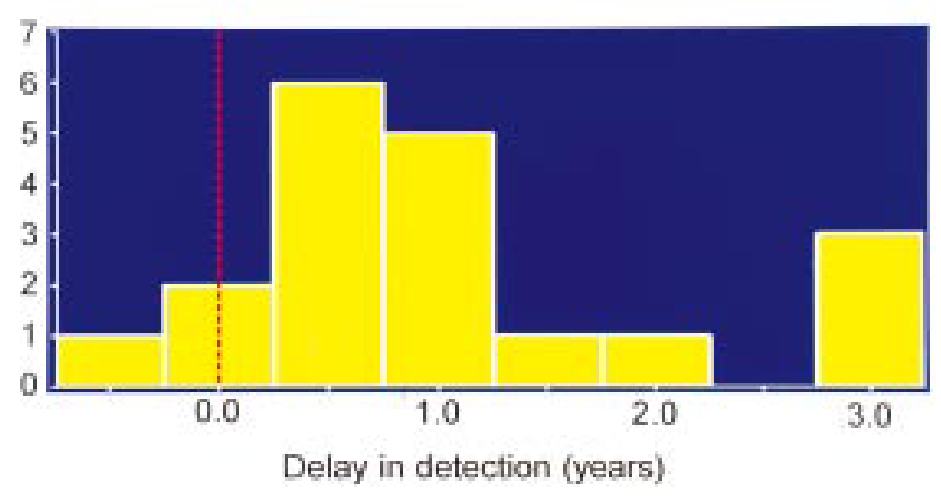

Figure 4 Histogram of delay in detection associated with STATPAC 2. The mean delay is 1.085 (SD 0.936) years.
Table 1 Detection times for PROGRESSOR and STATPAC 2

\begin{tabular}{lll}
\hline & \multicolumn{2}{l}{ Detection time (years) } \\
\cline { 2 - 3 } & Mean & $S D$ \\
\hline PROGRESSOR & 1.077 & 0.985 \\
STATPAC 2 & 2.161 & 1.357
\end{tabular}

$(\mathrm{p}<0.01$, Wilcoxon signed $\operatorname{rank} \mathrm{Z}$ test $)$

three fields was not applied for the final field of the series in this analysis: STATPAC 2 was judged as reliable if at least one location initially diagnosed as progressing was labelled with a black triangle in the single final field.

STATISTICAL ANALYSIS

For each field series, detection time (see above) was calculated for each algorithm. Detection times for PROGRESSOR were compared with their correlates for STATPAC 2 using a nonparametric test for paired data from two related samples (Wilcoxon signed rank $\mathrm{Z}$ test). ${ }^{30}$

Statistical analysis was performed using the software package sPss for Windows version 6.0.

\section{Results}

DETECTION TIMES

All 19 field series satisfied the progression criteria for both PROGRESSOR and STATPAC 2. The mean detection time for PROGRESSOR was 1.077 years with a standard deviation of 0.985 years. STATPAC 2 gave a mean detection time of 2.161 years with a standard deviation of 1.357 years. These findings are displayed in Table 1, and the individual differences between the algorithms for each field series are shown as a drop line graph in Figure 3. STATPAC 2 has consistently later detection times than PROGRESSOR: there is only one field series in which the detection time for PROGRESSOR is greater than that for STATPAC 2 .

DELAY

Delay in detection was calculated as the difference between the detection time for STATPAC 2 and that for PROGRESSOR. The mean delay in detection associated with STATPAC 2 was 1.085 years and the standard deviation was 0.936 years. As can be seen in Figure 4, the great majority of values of delay are greater than zero. In other words, PROGRESSOR detects progression sooner than STATPAC 2. This is borne out by the results of the Wilcoxon signed rank $Z$ test $(\mathrm{p}<0.01$, Table 2$)$.

Table 2 Comparison of detection times between PROGRESSOR and STATPAC 2 (Wilcoxon signed rank $Z$ test)

\begin{tabular}{lcc}
\hline & Cases & Mean rank \\
\hline Negative ranks & 1 & 3.00 \\
Positive ranks & 18 & 10.39 \\
Ties & 0 & \\
Total & 19 & \\
\hline$Z$
\end{tabular}

$Z=-5.1816, \mathrm{p}=0.0002$

Negative ranks occur when STATPAC 2 detects progression in a given field series earlier than PROGRESSOR.

Positive ranks occur when PROGRESSOR detects progression in a given field series earlier than STATPAC 2.

Ties occur when both algorithms detect progression in a given
Tien field series at the same time. 
RELIABILITY

For PROGRESSOR, at least one of the locations initially detected as progressing was still progressing in the final field of the series in $78.9 \%$ ( 15 out of 19$)$ of the field series. For STATPAC 2 , the corresponding figure was $68.4 \%$ (13 out of 19$)$.

\section{Discussion}

Previous work has shown that PROGRESSOR agrees closely with STATPAC 2 in terms of which test locations are classified as progressing and which are classified as stable. ${ }^{21}{ }^{25}$ Thus, it seems paradoxical that PROGRESSOR should be found to be superior to STATPAC 2 in the detection of progression. This apparent contradiction may be explained by considering the differences in methodology between this and the previous studies.

In the absence of a gold standard for glaucomatous visual field progression, the previous authors chose STATPAC 2 as an arbitrary gold standard against which to measure PROGRESSOR. STATPAC 2 was used to identify test locations which had 'unequivocally deteriorated' in the last three fields of series consisting of 16 fields each. ${ }^{25}$ The ability of PROGRESSOR to discriminate between these locations and the other locations (defined as stable by STATPAC 2) was then examined using the kappa statistic ${ }^{31}$ to assess the level of agreement with STATPAC 2 . A kappa coefficient of $\kappa=0.62(\mathrm{SE}=0.04)$ was obtained, which represents good agreement for this length of follow up. ${ }^{32}$ However, as the authors note, ${ }^{21} 25$ this analysis is inherently unable to detect any potential superiority of PROGRESSOR in detecting progression: it would be interpreted as a lack of specificity.

There are also theoretical explanations for the finding that PROGRESSOR detects progression sooner than STATPAC 2. The two algorithms attempt to diagnose progression in very different ways. STATPAC 2 compares each field under analysis with the baseline: information in fields after the baseline but before the field under analysis is ignored. STATPAC 2 is thus an event type of analysis and would be particularly sensitive to catastrophic, stepwise change. In contrast, PROGRESSOR uses all the fields up to and including the field under analysis: it is thus a trend type of analysis and would be more sensitive to gradual, sustained change. The pattern of pointwise change in progressive glaucoma has been investigated: although sudden, stepwise change does occur ${ }^{33}$ the mode of progression of the visual field of untreated normal tension glaucoma patients is best described by a pointwise linear analysis. ${ }^{24}$ Furthermore, STATPAC 2 is only able to classify locations if their level of loss can be determined relative to a normal database: previous work comparing PROGRESSOR with STATPAC 2 has excluded $26 \%$ of test locations from analysis because they cannot be classified by STATPAC $2 .{ }^{25}$

The normal tension glaucoma study group has reported that an event type of analysis can be used to diagnose visual field progression in a timely, sensitive and specific way. ${ }^{28}$ In this analysis, patients are followed every 3 months with visual field tests. If the criteria for suspected progression are met, the patient returns within 1-4 weeks for one or two confirmatory tests. If progression is confirmed in this way on two consecutive series of visits 3 months apart, true progression is diagnosed. Thus, the minimum time to diagnosis is 3 months and 1 week, which is earlier than for either algorithm in this study. The application of this testing protocol would enable earlier diagnosis of progression with STATPAC 2. However, PROGRESSOR would also be likely to detect change earlier in this case, since more frequent testing results in the earlier achievement of a significant slope: formal investigation would be required to determine whether PROGRESSOR or STATPAC 2 would benefit more from this strategy of confirmatory testing. Furthermore, this study protocol entails a high frequency of testing which would have profound resource implications if it were implemented on a routine basis, since it may involve three tests every 3 months.

In summary, this study was not designed to assess the sensitivity or specificity of PROGRESSOR or STATPAC 2 relative to an arbitrary gold standard (since this has been examined elsewhere ${ }^{1725}$ ), but rather to investigate the performance of the two algorithms in reliably detecting early change in a group of patients known to be deteriorating unequivocally on clinical grounds. The fact that PROGRESSOR consistently detected progression earlier than STATPAC 2, and detected it more reliably, suggests that PROGRESSOR is a useful software tool for the analysis of visual field progression in glaucoma.

Presented in part at the Association for Research in Vision and Ophthalmology annual meeting, 25 April 1996.

Supported in part by grants from the International Glaucoma Association and the Medical Research Council

The Institute of Ophthalmology retains the intellectual property rights to the PROGRESSOR software used in this study.

1 Boeglin RJ, Caprioli J, Zulauf M. Long-term fluctuation of the visual field in glaucoma. Am $\mathcal{F}$ Ophthalmol 1992; 113:396-400.

2 Flammer J, Drance SM, Zulauf M. Differential light threshold. Short- and long-term fluctuation in patients with glaucoma, normal controls, and patients with suspected glaucoma. Arch Ophthalmol 1984;102:704-6.

3 Zulauf M, Caprioli J. What constitutes progression of visual field defects? Sem Ophthalmol 1992;7:130-46.

4 Hoskins HD, Jensvold N, Zaretsky M, Hetherington J. Rate of progression of discrete areas of the visual field. In: of progression of discrete areas of the visual field. In: Kugler \& Ghedini, 1989:173-6.

5 Mikelberg FS. Do computerised visual fields and automated optic disc analysis assist in the choice of therapy in optic disc analysis assist in the
glaucoma? Eye 1992;6(Pt 1):47-9.

6 Hitchings K. Psychophysical testing in glaucoma. Br f OphHitchings K. Psychophysi

7 Johnson CA. Modern developments in clinical perimetry. Curr Opin Ophthalmol 1993;4:7-13.

8 Fitzke FW, McNaught AI. The diagnosis of visual field progression in glaucoma. Curr Opin Ophthalmol 1994;5:110-5.

9 Werner EB, Bishop KI, Koelle J, Douglas GR, LeBlanc RP, Mills RP, et al. A comparison of experienced clinical observers and statistical tests in detection of progressive visual field loss in glaucoma using automated perimetry. Arch Ophthalmol 1988;106:619-23.

10 Weber J, Koll W, Krieglstein GK. Intraocular pressure and visual field decay in chronic glaucoma. Ger $\mathcal{f}$ Ophthalmol 1993;2:165-9.

11 Smith SD, Katz J, Quigley HA. Analysis of progressive change in automated visual fields in glaucoma. Invest Ophchange in automated visual fields

12 Wegner A, Ugi I, Hofman A. A long-term visual field evaluation of glaucoma patients treated topically with timolol or 
carteolol. In: Mills RP, ed. Perimetry update 1992/1993. Amsterdam: Kugler \& Ghedini, 1993:143-5.

13 Holmin $C$ Kugler \& Ghedini, 1993.143-5. visual field in chronic glaucoma cases. A follow-up study using automatic perimetry. Acta Ophthalmol Copenh 1982;60:267-74

14 Wu D, Schwartz B, Nagin P. Trend analyses of automated visual fields. Doc Ophthalmol Proc Ser 1987;49:175-89.

15 O'Brien C, Schwartz B, Takamoto T, Wu DC. Intraocular pressure and the rate of visual field loss in chronic open-angle glaucoma. Am f Ophthalmol 1991;111:491500.

16 Chauhan BC, Drance SM, Douglas GR. The use of visual field indices in detecting changes in the visual field in glaucoma. Invest Ophthalmol Vis Sci 1990;31:512-20.

17 Birch MK, Wishart PK, O'Donnell N. Determining progressive field loss. In: Mills RP, Wall M, eds. Perimetry update 1994/1995. Amsterdam: Kugler \& Ghedini, 1995: update 1 .

18 Wild JM, Hussey MK, Flanagan JG, Trope GE. Pointwise topographical and longitudinal modeling of the visual field
in glaucoma. Invest Ophthalmol Vis Sci 1993;34:1907-16.

19 O'Brien C, Schwartz B. The visual field in chronic open angle glaucoma: the rate of change in different regions of the field. Eye 1990;4(Pt 4):557-62.

20 Heijl A, Lindgren G, Lindgren A, Olsson J, Asman P, Myers $\mathrm{S}$, et al. Extended empirical statistical package for evaluation of single and multiple fields in glaucoma: Statpac 2. In: Mills RP, Heijl A, eds. Perimetry update 1990/ 1991. Amsterdam: Kugler \& Ghedini, 1991:303-15.

21 Fitzke FW, Hitchings RA, Poinoosawmy D, McNaught AI, Crabb DP. Analysis of visual field progression in glaucoma. Br f Ophthalmol 1996;80:40-8.

22 Noureddin BN, Poinoosawmy D, Fitzke FW, Hitchings RA. Regression analysis of visual field progression in low tension glaucoma. Br f Ophthalmol 1991;75:493-5.
23 Poinoosawmy D, Wu J, Fitzke FW, Hitchings RA. Discrimination between progression and non-progression visual field loss in low tension glaucoma using MDT. In: Mills RP, field loss in low tension glaucoma using MDT. In: Mills RP, ed. Perimetry update

24 McNaught AI, Crabb DP, Fitzke FW, Hitchings RA. Modelling series of visual fields to detect progression in normal tension glaucoma. Graefes Arch Clin Exp Ophthalmol 1995; 233:750-5.

25 McNaught AI, Crabb DP, Fitzke FW, Hitchings RA. Visual field progression: comparison of Humphrey Statpac 2 and
pointwise linear regression analysis. Graefes Arch Clin Exp pointwise linear regression a

26 Werner EB, Adelson A, Krupin T. Effect of patient experience on the results of automated perimetry in clinically stable glaucoma patients. Ophthalmology 1988;95: $764-7$.

27 Werner EB, Krupin T, Adelson A, Feitl ME. Effect of patient experience on the results of automated perimetry in glaucoma suspect patients. Ophthalmology 1990;97:44-8.

28 Schulzer M. Errors in the diagnosis of visual field progression in normal-tension glaucoma. Ophthalmology 1994;101:1589-94.

29 Heijl A, Lindgren G, Olsson J. Normal variability of static perimetric threshold values across the central visual field. Arch Ophthalmol 1987;105:1544-9.

30 Altman DG. Practical statistics for medical research. 1st ed. London: Chapman and Hall, 1991.

31 Fleiss JL. Statistical methods for rates and proportions. New York: John Wiley, 1981:212-25.

32 Landis JR, Koch GG. The measurement of observer agreement for categorical data. Biometrics 1977;33:159-74.

33 Mikelberg FS, Drance SM. The mode of progression of visual field defects in glaucoma. Am f Ophthalmol 1984;98: 443-5. 